\title{
Análise da eficiência espectral para bandas licenciadas WiMAX considerando a duplexação e o perfil de tráfego
}

\author{
Márcia Maria Savoine \\ Norma Reggiani ${ }^{1}$ \\ David Bianchini ${ }^{1}$ \\ Omar Carvalho Branquinho ${ }^{1}$
}

\begin{abstract}
Resumo: Este trabalho apresenta a comparação, efetuada por meio de simulações de eventos discretos das técnicas de duplexação por divisão no tempo (TDD) e duplexação por divisão da frequência (FDD), dentro dos padrões da tecnologia WiMAx 3,5GHZ. O modelo de simulação considerou na fila de chegada dos serviços o conceito $F I F O$ e as simulações foram realizadas considerando cinco aplicações (Streaming, Download, Web, E-mail e Small-Transaction) para downlink e uplink. O parâmetro de eficiência utilizado foi o número mínimo de canais necessários em cada um dos canais padrões. A análise efetuada permitiu identificar que em tráfego de dados assimétricos a técnica TDD se apresentou mais eficiente e, observou-se existir ociosidade de espectro na técnica FDD.
\end{abstract}

Palavras-chave: Simulação. Técnicas TDD FDD. WiMAx.

\begin{abstract}
This work presents the comparison made by means of events discreet simulations the techniques of time division duplex (TDD) and frequency division duplex (FDD) within the standards of technology WiMAX 3,5GHz. The simulation model considered the concept FIFO in the services line arrival and the simulations were accomplished considering five applications (streaming, download, web, e-mail and small-transaction) for downlink and uplink. The regarded efficiency parameter was the minimum number of channels necessary in each one of the standard channels. The analyses were done considering, that in asymmetric data traffic, the TDD technique was more efficient and there were spectrum idleness in the FDD technique.
\end{abstract}

Keywords: Simulation. Techniques TDD FDD. WiMAx.

\footnotetext{
1 Mestrado Profissional em Gestão de Redes de Telecomunicações - Pontifícia Universidade Católica de Campinas, PUCCAMP, Caixa Postal 317, CEP: 13.012-970 - Campinas (SP) - Brasil

\{savoine, norma.reggiani, davidesperantista, omar.branquinho@gmail.com
}

http://dx.doi.org/10.5335/rbca.2012.2260

Revista Brasileira de Computação Aplicada (ISSN 2176-6649), Passo Fundo, v. 4, n. 2, p. 72-82, out. 201272 


\section{Introdução}

Ao final desta primeira década do segundo milênio, a demanda crescente para acesso onipresente de internet é uma exigência constante para serviços ao usuário final; seja ele doméstico, acadêmico, empresarial ou rural. A demanda de acesso para usuários residenciais envolve diversos aspectos da vida social, política e econômica, tais como entretenimento, educação, controle financeiro etc.

Da mesma forma, empresas necessitam de acesso rápido para transações comerciais em bancos ou empresas financeiras, e-mails com respostas de contratos e orçamentos, ou até mesmo para reuniões de videoconferência entre clientes, fornecedores, acionistas, funcionários e presidência. $\mathrm{Na}$ área rural, o acesso pode facilitar o conhecimento de informações estratégicas sobre agronegócios, envolvendo plantio, controle de doenças, pragas ambientais, controle de fluxo médico-veterinário, cotações em tempo real, entre outros. Em todos os ramos do conhecimento, essa demanda crescente de informação rápida e com qualidade impulsiona o acesso à internet em qualquer hora e lugar, indicando as redes wireless de longa distância como uma solução viável e eficiente.

Porém, prover banda-larga sem fio para dados, voz e vídeo não é ainda algo comum e frequente nas cidades brasileiras. Dentro desse quadro, uma das motivações do padrão IEEE 802.16, definido para redes de banda larga wireless, é oferecer acesso à internet sem fio, a longas distâncias, e com eficiência [1].

A tecnologia WiMAX, acrônimo de Worldwide Interoperability for Microwave Access, trata da interoperabilidade entre produtos baseados no padrão IEEE 802.16. O Institute of Electrical and Electronics Engineers (IEEE) é responsável pelo padrão IEEE 802.16 com o intuito de especificar formalmente redes semfio de banda larga, para cobrir grandes áreas metropolitanas, ou seja, redes Wireless Metropolitan Area Network (WMAN). Esse padrão possui soluções licenciadas e isentas de licenças, sendo a faixa de frequência para as primeiras de 2,5 GHz, 3,5 GHz e futuramente $10,5 \mathrm{GHz}$, e para as segundas a frequência de 5,8 GHz.

Conforme o IEEE 802.16, o WiMAx utiliza técnica de duplexação nos canais de comunicação, que consiste no processo de criação de canais bidirecionais para uplink e dowlink da transmissão de dados. Este padrão apresenta dois modos de duplexação, que pode ser tanto no tempo, duplexação por divisão no tempo, como em frequência, duplexação por divisão de frequência. Essa característica permite a interoperabilidade do IEEE 802.16 com sistemas celulares e outros sistemas sem fio, possibilitando, assim, uma flexibilidade de acesso para usuários.

A assimetria e simetria existentes nessas técnicas de comunicação são importantes para o WiMAX operando em 2,5 GHz e 3,5 GHz, pois preveem blocos de frequência de up e downlink para implementar FDD em canais distintos, mas permitem utilizar TDD nos blocos de subida e descida em um único canal, e o WiMAX $5,8 \mathrm{GHz}$ e $10,5 \mathrm{GHz}$ utilizam somente a técnica TDD em sua comunicação.

O objetivo deste trabalho é comparar a eficiência dos canais de comunicação no modo de duplexação TDD com o FDD, em banda licenciada WiMAX em 3,5GHz, simulando diversos cenários, envolvendo serviços em que estão presentes aplicações de streaming de vídeo, download, email, web e small-transaction. Pretende-se, desse modo, avaliar qual técnica tem maior eficiência em um tráfego de dados assimétrico, como é o caso da internet.

Neste artigo, as técnicas TDD e FDD são expostas e são mostradas as simulações que demonstram qual a melhor técnica a ser utilizada em determinados serviços, como download, web browsing, e-mail, streaming e small-transaction. Os resultados dessas simulações com FDD e TDD são discutidos e as conclusões são apresentadas na última sessão.

\section{FDD versus TDD}

A duplexação por divisão de tempo (TDD) utiliza uma mesma faixa de frequência para uplink e downlink, com um tempo-de-guarda entre eles, existindo uma competição de todos os transmissores pelo meio. Essa técnica está apresentada na Figura 2. 


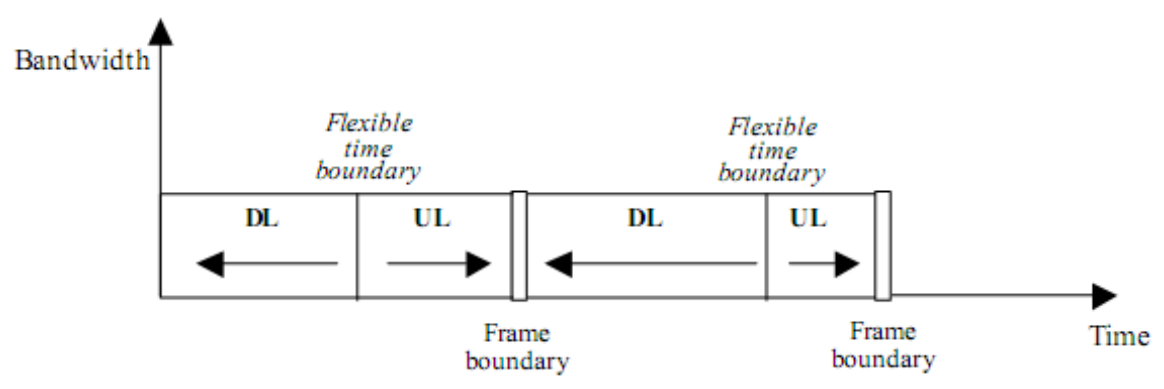

Figura 1: Sincronização TDD [2].

A técnica TDD distribui parte do canal para down e uplink e pode alocar dinamicamente a utilização do canal para down e uplink dependendo da quantidade de tráfego, provocando uma acomodação eficiente do canal para tráfego de dados por rajadas. Evidencia-se que, neste sentido, é acomodado o tempo de transmissão dedicado ao down e uplink. Essa transferência assimétrica é adequada para tráfego de internet, onde grandes quantidades de dados precisam trafegar pelo downlink.

Quando a taxa alocada entre uma parte do frame de downlink e uplink varia no tempo, a técnica de TDD é chamada dinâmica ou adaptável. A utilização de TDD adaptável em sistemas de acesso de rádio fixos possibilita um uso eficiente do espectro disponível, isso devido ao tráfego que é assimétrico e imprevisível e representa uma porcentagem considerável da carga de tráfego do sistema. A Figura 2 ilustra essa natureza assimétrica do TDD.

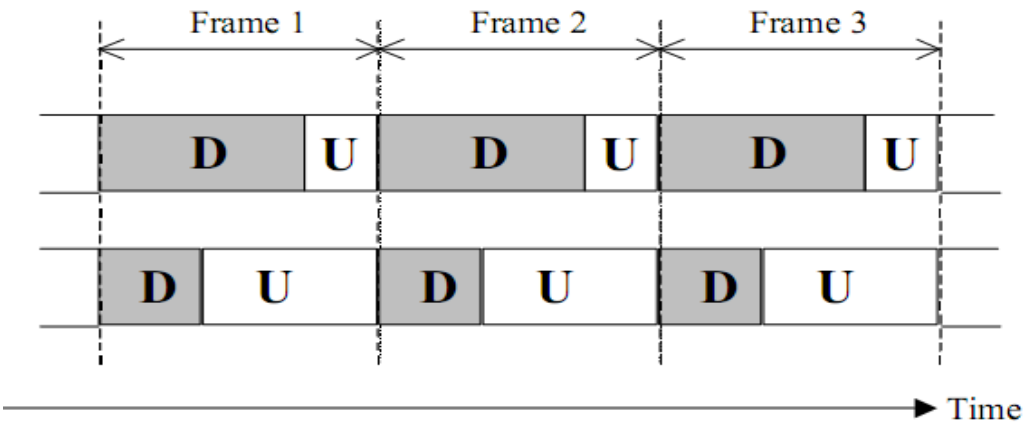

Figura 2: Frame de sincronização TDD de natureza assimétrica [2].

Devido à distribuição dinâmica de up e downlink, não há muito desperdício de espectro para operações assimétricas, ou seja, em serviços de última milha, onde o tráfego de uplink é tipicamente uma parte do tráfego de downlink. Pode-se observar que, reduzida a faixa do espectro, ainda é perdida nos tempos de guarda, mas é insignificante, comparado com o comprimento total de dados em uma faixa de tempo [3].

Por sua vez, a duplexação por divisão de frequência (FDD) requer um par de canais, um para transmissão e o outro para recepção, ou seja, um para downlink e outro para uplink. Qualquer canal duplex consiste, então, de dois canais simplex (um direto e outro reverso), contendo uma banda de guarda entre os dois canais para que não aconteça interferência. Esse espectro pareado permite a transmissão e recepção bidirecionais simultâneas e simétricas [2]. A Figura 3 ilustra um canal FDD.

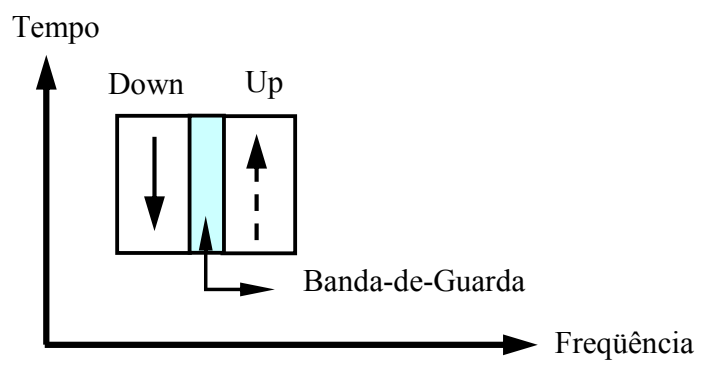

Figura 3: Utilização do espectro em FDD. [3] 
Os canais de comunicação FDD são sempre de tamanhos iguais, 50\% para downlink e 50\% para uplink. Uma banda de guarda de cerca de duas vezes o tamanho do canal de up e downlink é necessária para separar os canais de down e uplink. Isso equivale a uma perda adicional de 50\% no espectro [3].

Em aplicações de acesso à internet, de natureza assimétrica, isto é, quando a utilização da banda para downlink é muito maior do que a utilizada para uplink ou vice-versa, pode haver desperdício do espectro. Então, o modo de duplexação FDD é tipicamente utilizado em aplicações que requerem largura de banda igual em ambos os sentidos da comunicação, como as aplicações de voz ou videoconferência [4].

As técnicas de comunicação FDD e TDD são os dois esquemas de duplexação prevalecentes usados em redes banda-larga sem fios. O WiMAX pode empregar qualquer um deles para separar uplink e downlink nos sinais de comunicação. FDD é comumente utilizado para as aplicações que exigem uplink e downlink iguais na largura de banda. Já o TDD é frequentemente empregado na comunicação de cenários assimétricos [6].

\section{Cenários simulados}

O objetivo das simulações foi avaliar qual das técnicas, TDD ou FDD, é mais eficiente quando vários serviços estão disponíveis na rede. Foram feitas simulações com throughput médio de $600 \mathrm{kbps}$, tanto para a comunicação TDD como FDD, utilizando o software Arena ${ }^{\circledR}$ que gera o tempo das filas em minutos para os cenários propostos de TDD e FDD. O tempo das filas converge para um valor à medida que se aumenta $\mathrm{o}$ número de canais utilizado. A grandeza que caracteriza a eficiência dos padrões TDD e FDD é o número mínimo de canais necessário para que o tempo de espera de todos os serviços considerados tenha convergido para um valor fixo. Como canal significa alocação de banda no espectro de frequência, quanto menor este número, mais eficiente pode-se considerar a técnica de duplexação.

Cada tipo (i) de serviço será caracterizado pelos parâmetros:

- taxa média de chegadas $\left(\lambda_{i}\right)$;

- intervalos entre chegadas, descrito pela distribuição exponencial negativa, sendo: $\mathrm{f}(\mathrm{t})=\lambda \mathrm{e}^{-\lambda \mathrm{t}}$

- o tempo médio de serviço $\left({ }^{\tau_{i}}\right)$;

- número de canais;

- disciplina na fila: A disciplina da fila usado no modelo de simulação por eventos discretos deste trabalho utiliza o conceito FIFO (first-in, first-out), ou seja, o atendimento é feito por ordem de chegada, onde o primeiro a chegar é o primeiro a ser atendido.

A simulação por eventos discretos é própria para a análise de sistemas nos quais o estado discreto das variáveis muda apenas com a ocorrência de eventos (considerados instantâneos). Os modelos de simulação são analisados por métodos numéricos ao invés de métodos analíticos. Isso é, em vez de métodos analíticos que empregam o raciocínio dedutivo/matemático para resolver um modelo, consideram-se métodos numéricos que empregam procedimentos computacionais para executar os modelos matemáticos [7].

Determina-se que o Tempo Médio de Serviço $\tau_{i}$ é calculado pela expressão (1):

$$
\tau_{i}=\left[\left(\frac{(\text { Tamanho_do_Pacote })}{\text { Throughput }}\right) / 60 s\right]
$$

Onde:

- tamanho_pacote $(i)=$ tamanho do pacote por aplicação $(i)$;

- throughput = velocidade de transmissão (neste caso $600 \mathrm{Kbps}$ );

- $/ 60 \mathrm{~s}=$ divide-se por $60 \mathrm{~s}$, para se obter os valores em minutos.

Através do software de monitoramento NetPerSec ${ }^{\circledR}$ ou observações estatísticas, pode-se constatar que o tráfego gerado através do canal de comunicação pelos usuários é mais intenso em determinados horários e menos intenso em outros [5].

Revista Brasileira de Computação Aplicada (ISSN 2176-6649), Passo Fundo, v. 4, n. 2, p. 72-82, out. 201275 
Esses períodos de maior movimento (PMM) dos usuários, também conhecidos como horários de maior movimento (HMM) ou busy hour (BH) são considerados como base para o cálculo da taxa de chegada no simulador de eventos discretos. Sendo:

$$
\lambda_{i}=\left(\frac{N^{\circ}-\text { de }-\operatorname{Arquivos}(i)}{\operatorname{Horas}(i) * 60 \min }\right)
$$

Onde:

- $\lambda_{i}=$ Taxa de Chegada de cada Aplicação em particular;

- $\mathbf{n}^{\circ}$-de-arquivos $(i)=$ quantidade de arquivos de cada aplicação que compõem a BH;

- horas $(i)=$ quantidade de tempo que compõem a BH;

- *60min = multiplica-se por 60min, para se obter os valores em minutos.

Como citado, a Busy-Hour (BH) é considerada como base para cálculo da taxa de chegada no simulador em todas as aplicações. Tem-se então a Tabela 1 mostrando todos os serviços, com todas as variáveis envolvidas.

Onde para todos os serviços é considerado um Percentual de Atividade na Sessão ${ }^{2}$ de um valor $(i)$, será aberto uma Sessão de Terminal de Leitura ${ }^{3}$, com um valor $(i)$ e, onde a interface área colocará em estado de dormant $^{4}$ a portadora em $(i)$ segundos.

Percebe-se que os serviços Streaming e Small-Transaction ocuparão a interface aérea 100\% do tempo; isto ocorre devido ao tempo-de-leitura ser menor que o tempo de dormant.

Tabela 1: Modelo de Tráfego para um usuário e percentual de atividade na sessão

\begin{tabular}{l|c|c|c}
\hline \multicolumn{1}{c|}{ Serviço } & $\begin{array}{c}\text { Tempo de Leitura } \\
\text { (em segundos) }\end{array}$ & $\begin{array}{c}\text { Quantidade de } \\
\text { Utilização na } \\
\text { Sessão }\end{array}$ & $\begin{array}{c}\text { Percentual de } \\
\text { Atividade na } \\
\text { Sessão (\%) }\end{array}$ \\
\hline Streaming & $\mathrm{n} / \mathrm{a}$ & 1 & $100 \%$ \\
\hline Download & 180 & 1 & $60 \%$ \\
\hline Web & 40 & 20 & $36 \%$ \\
\hline E-mail & 60 & 10 & $18 \%$ \\
\hline Small-Transaction & 5,5 & 2 & $100 \%$ \\
\hline
\end{tabular}

Salienta-se que os serviços considerados foram de streaming, download, web, e-mail e small-transaction, com as seguintes características [5], para cada um deles, conforme Tabela 2.

Tabela 2: Característica dos serviços utilizados

\begin{tabular}{l|c|c}
\hline \multicolumn{1}{c|}{ Serviço } & Número de arquivos/tempo & Tamanho do pacote \\
\hline Streaming & $0,533 \mathrm{~min} /$ imagem & $2.400 \mathrm{kbytes}$ \\
\hline Download & $0,4444 \mathrm{~min} /$ arquivo & $16.000 \mathrm{kbits}$ \\
\hline Web & $0,012 \mathrm{~min} /$ pacote & $432.000 \mathrm{bits}$ \\
\hline E-mail & $0,0022 \mathrm{~min} /$ pacote & $80.000 \mathrm{bits}$ \\
\hline Small-transaction & $0,000115 \mathrm{~min} /$ pacote & $4.160 \mathrm{bits}$ \\
\hline
\end{tabular}

2 Percentual de atividade na sessão: correspondente à parte do tempo total em que o usuário ficou realmente ativo.

3 Sessão de terminal de leitura: é o tempo compreendido entre duas atividades realizadas pelo usuário na rede para uma determinada aplicação $(i)$. Utilizado normalmente para leitura e uso das aplicações descarregadas (download), medidos em segundos.

4 Dormant: situação onde o usuário com sessão aberta não esteja transacionando dados por um intervalo de tempo (baixa atividade), entra em estado dormente. 
Foram utilizados três cenários para as simulações em ambas as técnicas de duplexação, onde a variação dos cenários ocorreu de acordo com o perfil de tráfego utilizado:

- $1^{\circ}$ cenário: tráfego de dados simétrico; ou seja, considerando o mesmo perfil de tráfego para downlink e uplink;

- $2^{\circ}$ cenário: tráfego de dados igualmente assimétrico para todos os serviços; ou seja, considerando o tráfego de downlink é 2, 5, 10 e 15 vezes maior que o de uplink;

- $3^{\circ}$ cenário: tráfego de dados com assimetrias diferenciadas por serviços; ou seja, considerando o tráfego de downlink nos diversos serviços variando de 2 a 5 vezes maior, de 5 a 10 vezes maior, e de 10 a 15 vezes maior que o uplink e de 2, 5, 10 e 15 vezes mais no downlink que o uplink para os serviços de streaming e small-t.

A Figura 4 ilustra esses três cenários acontecendo na interface área em ambas as técnicas de duplexação.

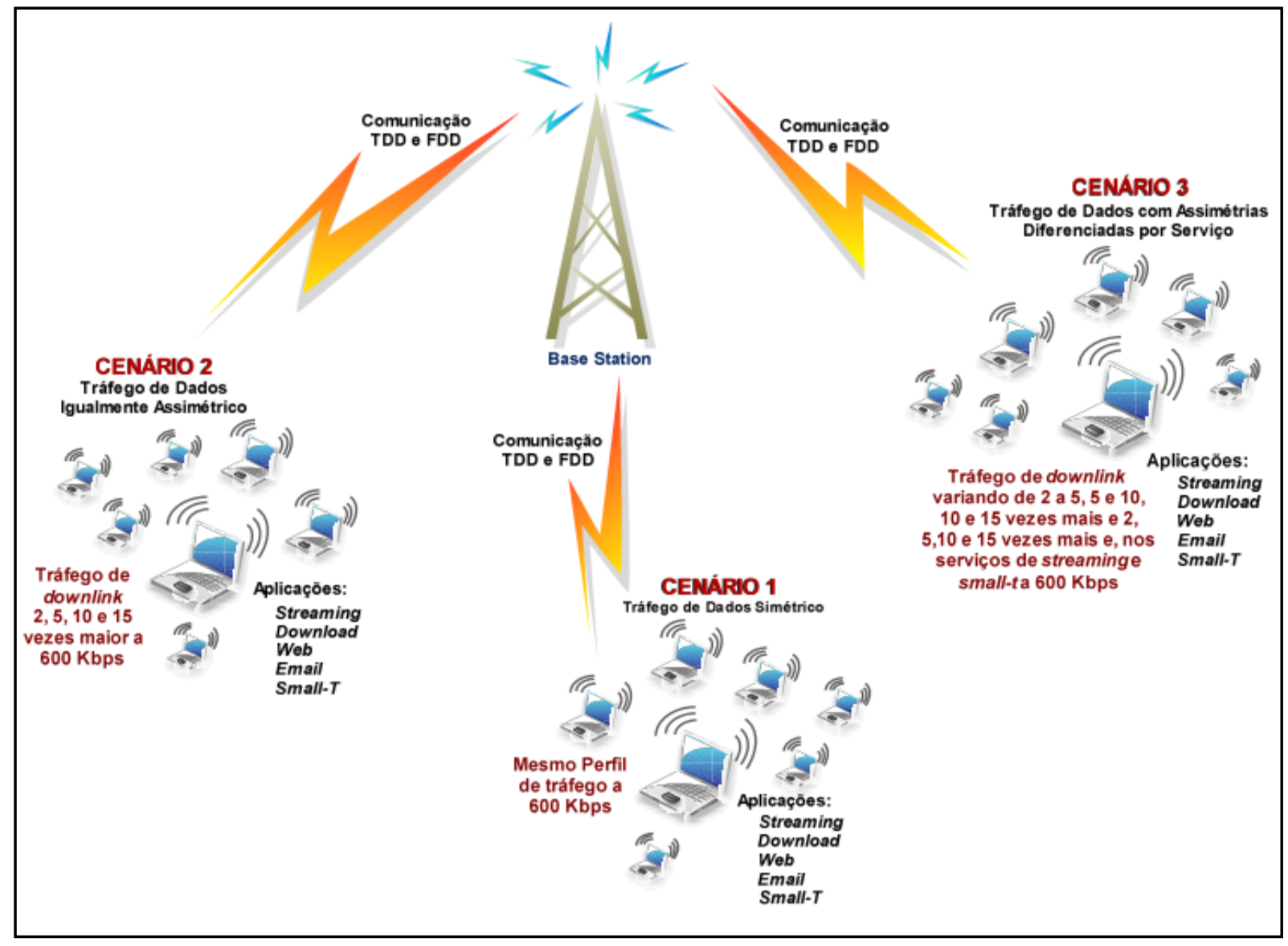

Figura 4: Ambiente com todos os cenários das simulações nas comunicações TDD e FDD.

\section{Simulações das aplicações em canais com duplexação TDD e FDD}

Para as simulações, foi considerado que a base station (BS) controlará as estações móveis (AT) e será vista como centro de controle [5]. A área de cobertura da BS de downlink e uplink é considerada para nove usuários utilizando cinco serviços (Streaming, Download, Web, E-mail e Small- Transaction) dos provedores de serviço da internet. 
O modelo de simulação é baseado em eventos discretos, onde o tipo de filas das chegadas dos serviços utiliza o conceito FIFO. A simulação foi desenvolvida utilizando-se do software Arena, podendo gerar o tempo das filas em minutos para os cenários propostos TDD e FDD.

\subsection{Cenário 1 - Tráfego simétrico}

Nesse cenário foram considerados os cinco serviços (streaming, download, web, e-mail e smalltransaction) com o mesmo tráfego tanto para downlink e uplink, sendo que o número de canais na BS varia de 4 a 60, com um throughput de $600 \mathrm{kbps}$, em ambos os modos. A Figura 5 ilustra essa situação mostrando a igualdade da quantidade de canais ocupados em ambas as técnicas.

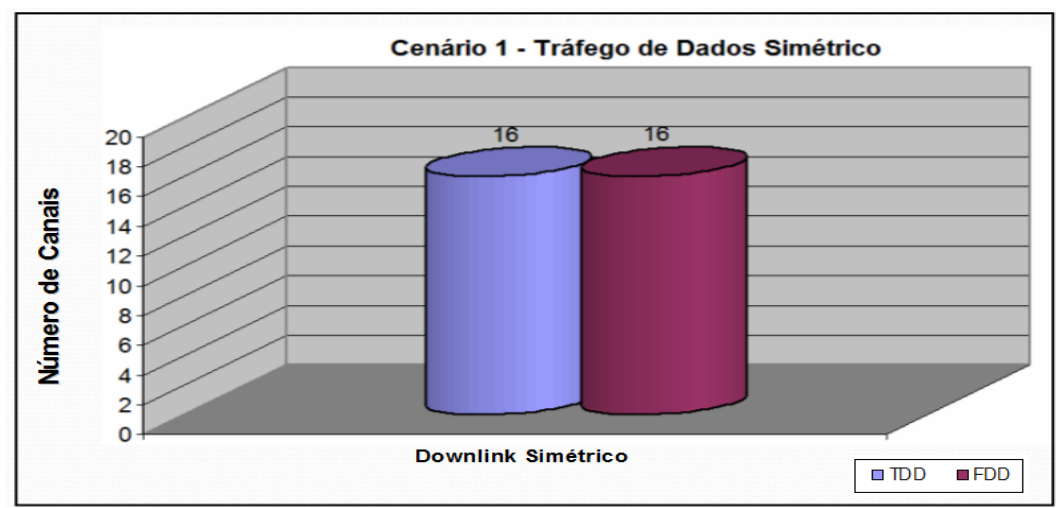

Figura 5: Cenário 1- Resultados das técnicas TDD e FDD com tráfego simétrico para todos os serviços

\subsection{Cenário 2 - Tráfego igualmente assimétrico para todos os serviços}

Nesse cenário consideram-se também os cinco serviços (streaming, download, web, e-mail e smalltransaction) tanto para downlink e uplink, sendo que o número de canais na BS varia de 4 a 60 e o throughput continua de $600 \mathrm{kbps}$. O perfil de tráfego para downlink, nesse caso, é igualmente maior do que para uplink para todos os serviços. Considerou-se a taxa de chegada dos serviços para downlink 2, 5, 10 e 15 vezes maior do que para uplink, em ambas as técnicas. A Figura 6 ilustra os resultados obtidos, que apontam para a conveniência da técnica TDD frente à FDD nesse cenário, pois quanto maior é a diferença entre o tráfego de down e uplink, maior é o número de canais necessário na técnica FDD comparativamente à técnica TDD.

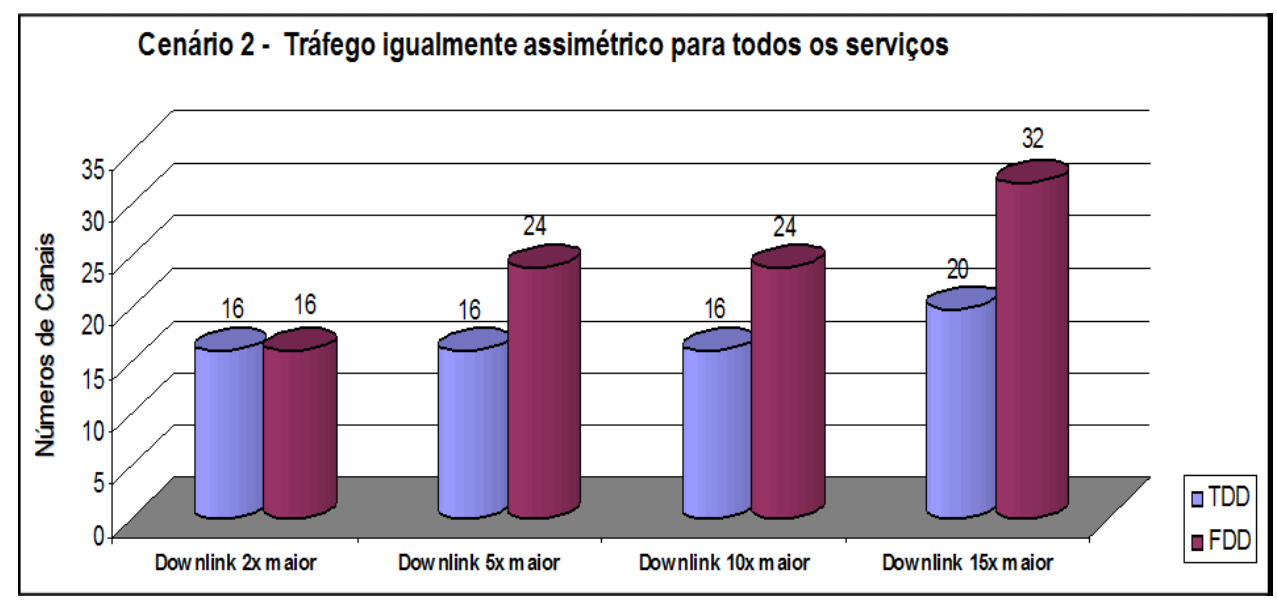

Figura 6: Resultados das técnicas TDD e FDD com tráfego igualmente assimétrico para todos os serviços

\subsection{Cenário 3 - Tráfego assimétrico de modo diferenciado por serviços}

$\mathrm{O}$ cenário 3 se desdobra em quatro estudos. No primeiro cenário, 3A, o tráfego é assimétrico e diferenciado por serviços. Nesse caso a assimetria foi feita considerando-se, para cada serviço, duas situações: (a) que esse serviço tenha o downlink $2 x$ maior que o uplink, enquanto os outros serviços ficam com o downlink 
5x maior que o uplink; (b) que este serviço tenha o downlink 5x maior que o uplink, enquanto os outros serviços apresentem o downlink 2x maior que o uplink. Nesse cenário, identificaram-se duas situações nas quais a técnica TDD mostrou melhor desempenho, mas, em todas as demais situações, a performance da técnica TDD foi equivalente à da técnica FDD. Ou seja, ao se ter um tráfego maior com os serviços de download e web, a técnica FDD é impulsionada a utilizar uma maior quantidade de canais, dando indícios de não conseguir gerenciar esses dois serviços de maneira adequada; pois são serviços que o tempo de dormant na interface aérea é maior que o tempo de leitura que é utilizada por eles, trazendo, nesse sentido, uma demora em sua execução. Percebe-se que a técnica FDD não consegue gerenciar de maneira satisfatória um tráfego assimétrico, isso porque a alocação do canal é constante e a faixa-de-tempo são iguais, características adequadas para tráfego de dados simétrico. A Figura 7 mostra os resultados do cenário 3A em ambas as técnicas. Essas características da técnica FDD tornamse cada vez mais evidentes, com todos os serviços, nos cenários 3B (Figura 7), 3C (Figura 8) e 3D (Figura 9), onde a assimetria do tráfego é crescente.

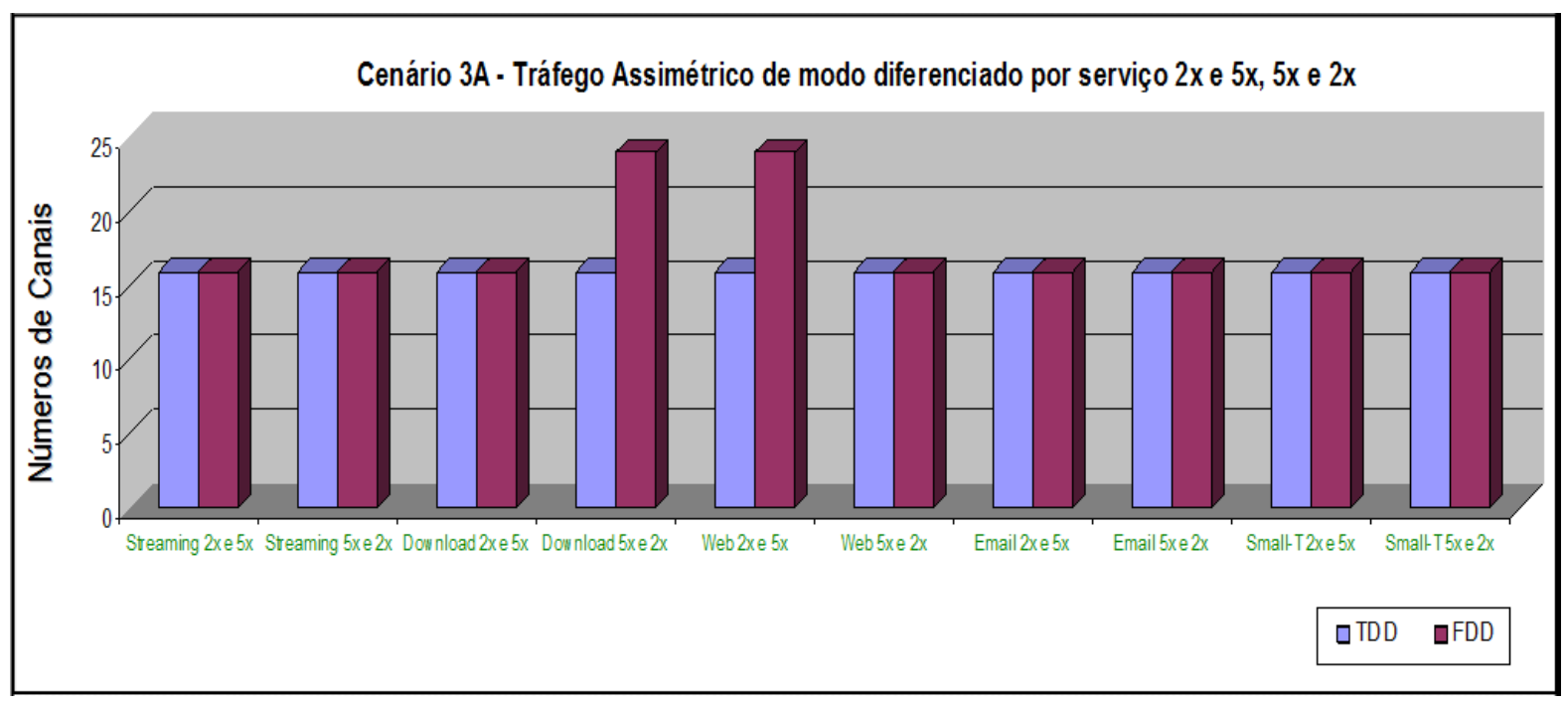

Figura 7: Cenário 3A - Resultados das técnicas FDD e FDD diferenciado por serviços

No cenário 3B, onde se mantêm o tráfego assimétrico diferenciado por serviço do mesmo modo que no cenário 3A, a variável consiste no fato de que o acréscimo de downlink com relação ao uplink é realizado de 5 e 10 vezes. Neste estudo, já se torna perceptível uma melhor utilização dos canais com a técnica TDD e uma maior ociosidade de espectro com a técnica FDD. Os serviços que utilizam mais canais com a técnica FDD são download (em ambas as situações), web e email (estes somente na situação de 5x e 10x). Essa situação é demonstrada na Figura 8.

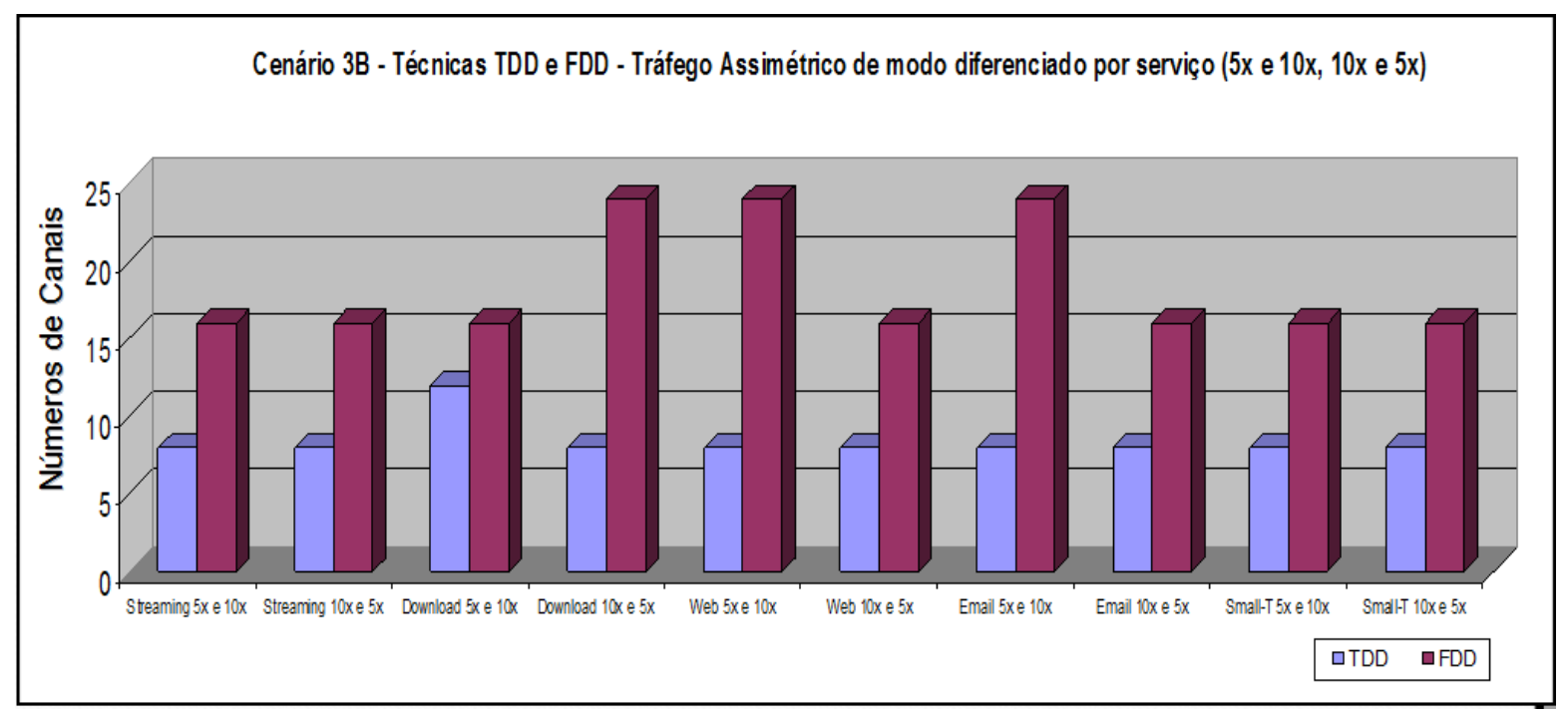

Revista Brasileira de Computação Aplicada (ISSN 2176-6649), Passo Fundo, v. 4, n. 2, p. 72-82, out. 201279 
Figura 8: Cenário 3B: Resultados das técnicas TDD e FDD diferenciado por serviços

Já no cenário 3C, as condições de tráfego são mantidas, sendo que o fator de acréscimo de downlink para uplink se faz de 10 e 15 vezes. Neste estudo, verifica-se que há uma melhor utilização dos canais com a técnica TDD, havendo uma grande ociosidade na utilização do espectro com a técnica FDD. Os serviços que consomem mais canais com a técnica FDD nesse cenário são: streaming, download, email e small-t (todos os três primeiros serviços com a situação 10x e 15x, e o último somente na situação de 15x e 10x). A Figura 9 ilustra esse resultado.

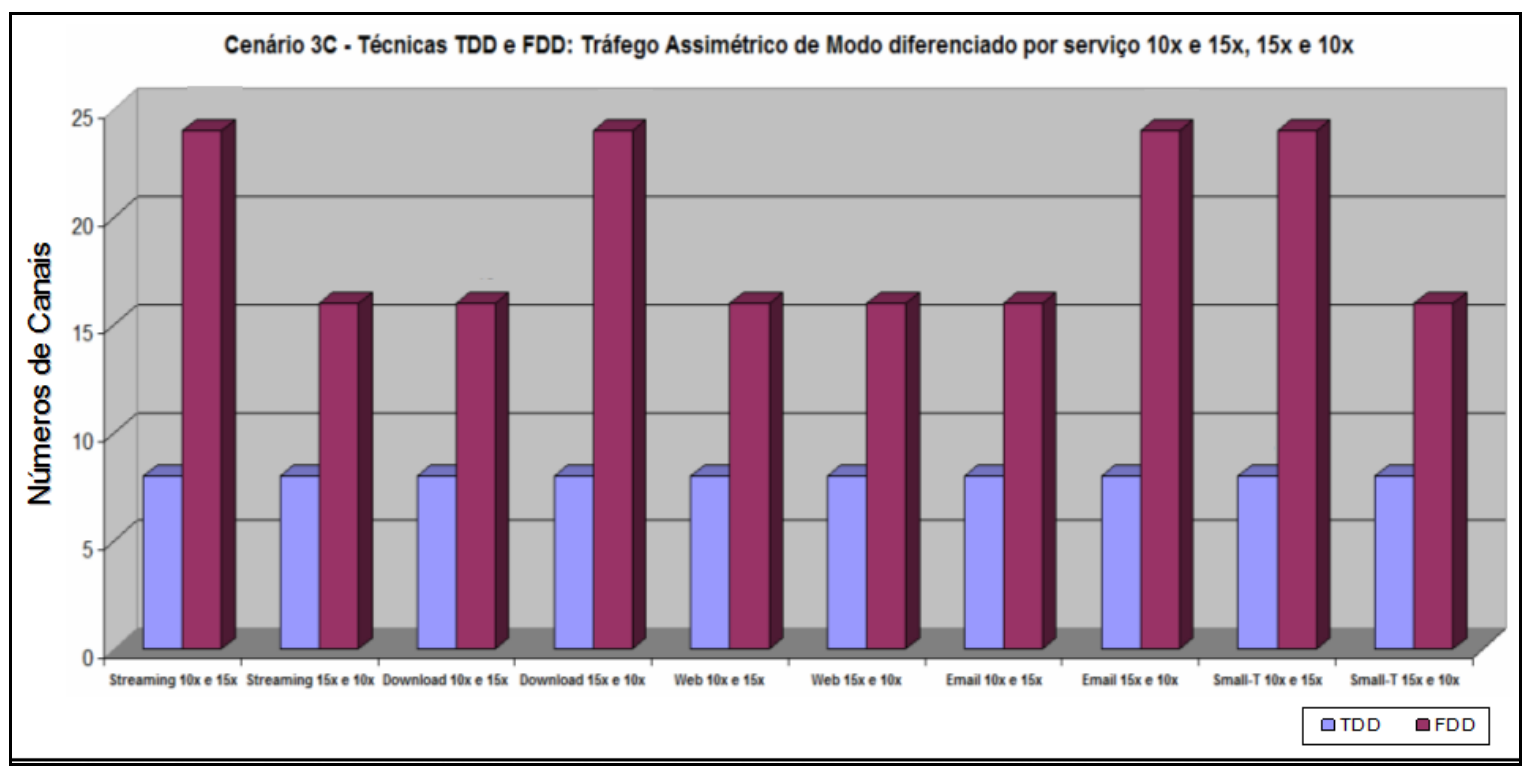

Figura 9: Cenário 3C: Resultados das técnicas TDD e FDD diferenciado por serviços

Nesse último estudo, o cenário 3D tem o tráfego também assimétrico e diferenciado pelos serviços de streaming e small-t, sendo realizado na proporção de 2x, 5x, 10x e 15x. Identifica-se uma utilização melhor dos canais com a técnica TDD que, embora muito pequena para ser impactante, mas mesmo assim, apresenta melhor resultado com estes serviços que necessitam de mais espectro. Constata-se que existe ociosidade do espectro com a técnica FDD. O serviço que utiliza mais canais com a técnica FDD neste cenário é small-t, Figura 10.

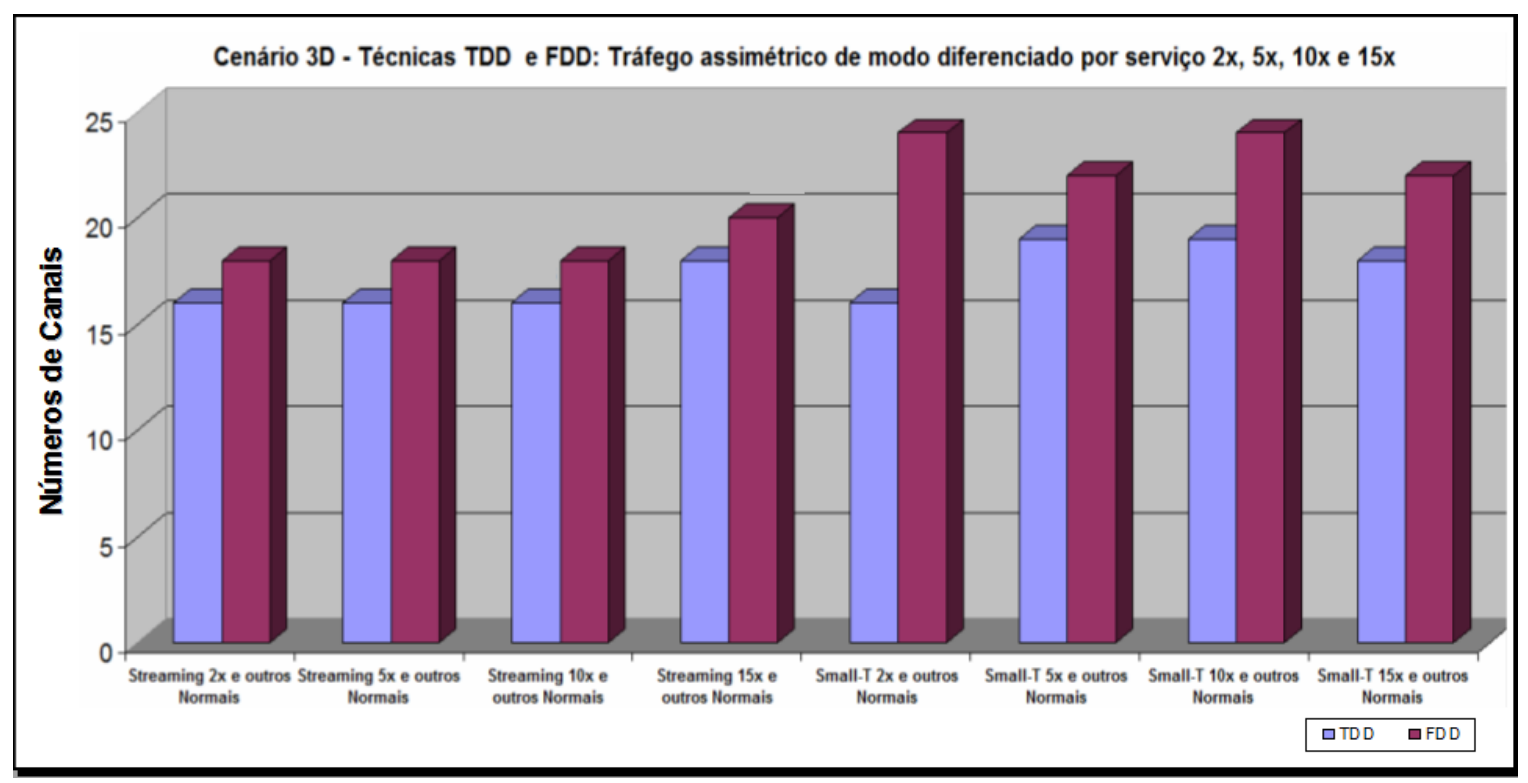

Figura 10: Cenário 3D: Resultados das técnicas TDD e FDD diferenciado por serviços 
Para análise dos resultados em todos os cenários simulados, sendo simétrico e assimétrico, tem-se uma variação na quantidade de canais. Analisando o Gráfico 7, pode-se perceber que, em um tráfego de dados simétrico (cenário 1) as duas técnicas mostram-se equivalentes. Já nos cenários 2 e 3, sendo um tráfego de dados assimétrico, a técnica TDD se mostra mais eficiente. É possível concluir-se que, ao se ter um volume de tráfego de dados intenso e assimétrico (como exemplo, o tráfego de dados da internet), a técnica TDD mostrará maior eficiência do que a FDD; ou ainda, que a ociosidade na técnica FDD tenderá a aumentar com o crescimento do tráfego de dados.

Pode-se perceber ainda, pela Figura 11, que a diferença entre as técnicas variam de 8 a 16 canais no cenário 2 e, de até 16 canais no cenário 3. Podendo, sim, esses valores, serem indicadores importantes na escolha de utilização das técnicas.

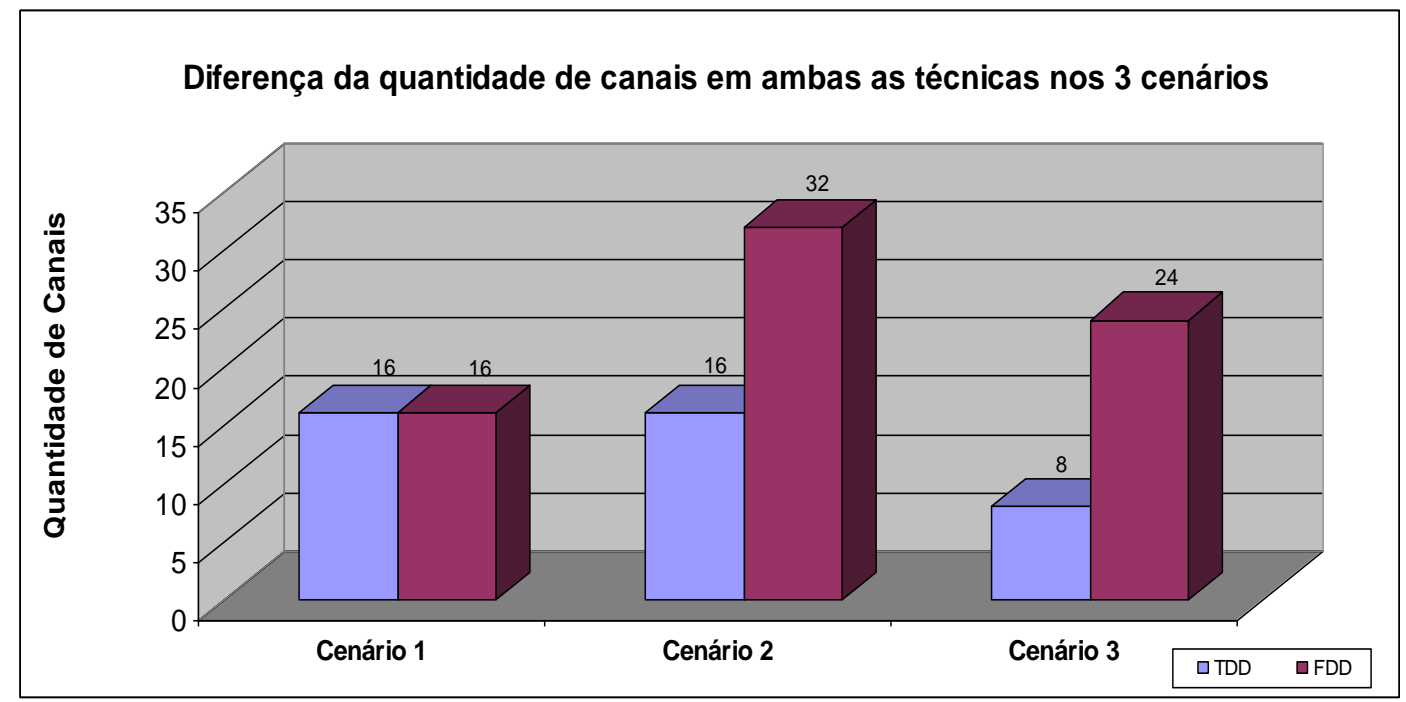

Figura 11: Diferença da quantidade de canais em ambas as técnicas nos 3 cenários

\section{Conclusão}

O WiMAX é uma das mais promissoras tecnologias mundiais para prover acesso banda-larga sem fio a longas distâncias. E por esse motivo, para prover acesso a redes com qualidade, é importante fazer a avaliação da eficiência espectral dessa tecnologia.

A eficiência do espectro é um parâmetro fundamental e extensamente usado para comparar tecnologias sem fios diferentes ou avaliar a eficiência de alguma tecnologia em específico.

Em redes sem fio banda-larga as técnicas de duplexação TDD e FDD são as mais utilizadas e o WiMAX pode empregar qualquer uma das duas técnicas ${ }^{5}$. Ambas, FDD e TDD, têm suas próprias vantagens, dependendo das aplicações. A técnica FDD utiliza canais distintos de frequência onde são atribuídas a uplink e downlink. Devido à natureza simétrica, os canais de transmissão de up e downlink da técnica FDD são sempre de tamanho igual. Consequentemente, FDD é utilizado para as aplicações que requerem largura de banda iguais de up e downlink.

\footnotetext{
5 Existe uma técnica híbrida de duplexação denominada HFDD - FDD Half-duplex. A técnica HFDD é bem parecida com a TDD. Um dispositivo de HFDD transmite e recebe em tempos diferentes como um dispositivo TDD. A diferença é que também usa frequências diferentes para transmitir e receber ao comunicar com uma Base Station FDD.

Outra técnica de duplexação existente refere-se ao TDD, isso acontece quando a relação entre os intervalos de tempo designados para down e uplink varia no tempo. Nesse caso, a técnica TDD é chamada ATDD - TDD dinâmico ou adaptável.
} 
Em contrapartida, TDD usa uma única frequência para transmitir sinais de ambas as direções, uplink e downlink. TDD divide o fluxo de dados em quadros e atribui diferentes faixas de tempo para as transmissões em up e downlink, dentro de cada frame. Considerando que os dispositivos usam o mesmo canal de frequência para transmitir ou receber sobre demanda, de forma flexível, a técnica TDD pode controlar a quantidade de largura de banda atribuída ao up e downlink. Por essa razão, TDD tem maior eficiência na utilização do espectro nos cenários de comunicação assimétricos que o FDD, onde o tráfego de uplink poderia ser menor que o tráfego downlink.

Neste trabalho foi analisada a eficiência espectral considerando estas técnicas de duplexação citadas, através de simulações com um perfil e tráfego de dados simétrico e assimétrico; dentro dos padrões da tecnologia WiMAX 3,5 GHz.

As simulações realizadas com o software Arena ${ }^{\circledR}$ permitiram uma análise das cinco aplicações: streaming, download, web, email e small-t, com as técnicas FDD e TDD, avaliando o tempo médio de serviço. Os resultados mostraram que em um tráfego de dados simétrico as duas técnicas são equivalentes e, em um tráfego de dados assimétrico, a técnica TDD mostra-se mais eficiente indicando um melhor desempenho e, uma ociosidade de espectro é apresentada na FDD.

Se considerar o tempo de guarda na técnica TDD, aumentará a quantidade de canais em torno de $10 \%$, o que representaria uma pequena desvantagem na diferença de número de canais, porém, não invalidaria os resultados aqui encontrados que apontam para o melhor desempenho da técnica TDD.

Percebe-se então que TDD tem grande vantagem ao gerenciar a variação de tempo de gráfico de up e downlink, permitindo um ganho de capacidade em relação ao FDD.

Como continuidade deste trabalho pretende-se realizar outras simulações com throughput médio de 900 Kbps, que proverá outras situações que possibilitem perceber a quantidade de canais necessários, bem como, quais serviços serão beneficiados. Ou ainda, pode-se conceber um controle de serviços para clientes com possíveis taxas melhores.

Outra possível continuação consiste em proceder a uma análise quantitativa deste trabalho, pois o mesmo trata-se de uma análise qualitativa. Isso pode ser feito separando os diversos serviços e tratando-os estatisticamente de forma diferenciada. Foi utilizada a distribuição exponencial negativa para todos os serviços aqui utilizados, sendo que, para refinar esse modelo, seria importante utilizar distribuições diferentes em cada aplicação. Então, poderá ser realizada uma análise diferenciada priorizando-se o atendimento aos serviços.

\section{Referências}

[1] SAVOINE, M.M.; REGGIANI, N.; BRANQUINHO, O.C.; BIANCHINI, D.; ARRUDA, W.C. Avaliação da Duplexação no WiMAX 3,5 GHz Baseado no Perfil de Tráfego. SBPO, 2008a.

[2] GRONDALEN, O.; et. al. Time Division Duplex- Flexible and efficient for milimetre broadband access systems. EMBRACE, 2002.

[3] PROXIM, Wireless Corporation. Advantages of Time Division Duplex (TDD) for Broadband Wireless in Last-Mile Applications. Position Paper, 2006.

[4] SAVOINE, M.; et al. Análise dos Métodos de Duplexação em Banda Licenciada WiMAX. MOMAG, $2008 \mathrm{~b}$.

[5] MENON, J.M. Dimensionamento de Tráfego de Rede de Dados para Sistema Celular 1XEV-DO. MOMAG, 2007.

[6] RONG B.; et. al. Downlink Call Admission Control in Multiservice WiMAX Networks. IEEE, ICC, 2007.

[7] MIYAGI, P.E. Introdução a Simulação Discreta. Escola Politécnica da Universidade de São Paulo. Departamento de Engenharia Mecatrônica e de Sistemas Mecânicos. São Paulo, 2004. 